TU-769

May, 2006

\title{
QCD Correction to Neutralino Annihilation Process and Dark Matter Density in Supersymmetric Models
}

\author{
Takeo Moroi, Yukinari Sumino and Akira Yotsuyanagi \\ Department of Physics, Tohoku University, Sendai 980-8578, JAPAN
}

\begin{abstract}
We calculate QCD correction to the neutralino annihilation cross section into quark anti-quark final state and discuss its implications to the calculation of neutralino relic density. We see that the QCD correction enhances the pair-annihilation cross section by $O(10 \%)$ when final-state quarks are non-relativistic. Consequently, when the lightest neutralinos dominantly annihilate into a $t \bar{t}$ pair, the relic density of the lightest neutralino is significantly affected by the QCD correction, in particular when the lightest-neutralino mass is close to the top-quark mass.
\end{abstract}




\section{Introduction}

Low energy supersymmetry has been regarded as an attractive candidate of new physics since it can solve various problems in the standard model of particle physics. One of the strong motivations of supersymmetry is that it provides a plausible candidate of cold dark matter. Recent precise cosmological observations have provided strong evidence of the existence of dark matter. In particular, Wilkinson Microwave Anisotropy Probe (WMAP) experiment has determined the present density parameter of the dark matter, which is given by [1]

$$
\Omega_{\mathrm{c}} h_{100}^{2}=0.105_{-0.013}^{+0.007} \quad(68 \% \text { C.L. })
$$

where $h_{100}$ is Hubble constant in units of $100 \mathrm{~km} / \mathrm{sec} / \mathrm{Mpc}$. From the particle-physics point of view, however, this raises a serious question since there is no viable candidate of dark matter in the particle content of the standard model. In supersymmetric models, the lightest superparticle (LSP) is stable due to $R$-parity conservation and hence it can be a candidate of cold dark matter. It has been widely recognized that, if the lightest neutralino $\chi_{1}^{0}$ is the LSP, its thermal relic density can become consistent with the WMAP value given in Eq. (1.1) in some parameter region. For a better understanding of the evolution of the universe, it is important to confirm or exclude the idea of neutralino (or LSP) dark matter.

The first important step of such test is to find low-energy supersymmetry in future collider experiments. Once superparticles are found, in addition, it is also important to quantitatively test the idea of neutralino dark matter. Quantitative test will be performed by calculating the relic density of neutralino, using mass and coupling parameters of superparticles measured in collider experiments [2]. Currently, cosmological observations have determined the dark matter density with an accuracy of $O(10 \%)$, hence the density parameter of the neutralino $\Omega_{\chi_{1}^{0}}$ is desired to be calculated theoretically at the same (or better) accuracy.

As is well known, the relic density of neutralino is sensitive to the neutralino pair annihilation cross section, which strongly depends on mass and mixing parameters of the superparticles. $\# 1$ Thus, for a precise calculation of the relic density, detailed calculation of the pair annihilation cross section is necessary. Indeed, in the case where the lightest neutralino is the LSP, the pair annihilation cross section and relic density of the lightest neutralino have been calculated in detail, in particular in connection with the WMAP observation [3].

So far, most of the previous calculations of pair annihilation cross section have been based on tree-level calculation. (See, however, 4.) One may naively estimate the size of one-loop corrections to be $O\left(\lambda^{2} / 16 \pi^{2}\right)$ with $\lambda$ being relevant coupling constant, hence it is $O(1 \%)$ or smaller as far as $\lambda \lesssim 1$. In such a case, radiative corrections to the pair annihilation cross section may be unimportant for the purpose of calculating $\Omega_{\chi_{1}^{0}}$ with an accuracy of $O(10 \%)$. If we consider QCD correction, however, this is not always the case. If we consider processes with $q \bar{q}$ final state (with $q$ and $\bar{q}$ being quark and anti-quark, respectively), QCD correction becomes $O(10 \%)$ when final-state quarks are non-relativistic. In the so-called "focus-point"

\footnotetext{
${ }^{\# 1}$ In some case, coannihilation process also plays an important role. In this paper, however, we assume that the mass of the lightest neutralino is quite far from the second-to-the-lightest superparticle, and that the coannihilation process is ineffective.
} 
model [5], for example, the annihilation process into a top quark pair, $\chi_{1}^{0} \chi_{1}^{0} \rightarrow t \bar{t}$, becomes one of the dominant pair annihilation processes [6, 7]. Then, QCD correction becomes important when the lightest neutralino mass is close to the top-quark mass due to threshold enhancement of the QCD correction. (The threshold enhancement is well known e.g. from the analysis of the cross section for $e^{+} e^{-} \rightarrow t \bar{t}$ close to $t \bar{t}$ threshold [8]).

The main purpose of this paper is to calculate the next-to-leading order (NLO) QCD correction to the neutralino annihilation cross section into $q \bar{q}$ final state and to discuss its implications to the calculation of the relic density of the lightest neutralino. (In fact, we also have to consider real gluon emission to take care of infrared divergences. Thus, we consider processes of the type $\chi_{1}^{0} \chi_{1}^{0} \rightarrow q \bar{q}(g)$, with $g$ being the gluon.) As we will see, QCD correction may become $O(10 \%)$. Since the relic density of the lightest neutralino is approximately inversely proportional to pair annihilation cross section, this has certain significance for a precise calculation of the dark matter density.

In addition, for scenarios where one of very weakly interacting superparticles (like gravitino [9], axino [10, or right-handed sneutrino [11, which are recently called superWIMPs) is the LSP, study of the QCD correction has some significance. In such scenario, the lightest neutralino becomes unstable and decays into a superWIMP (and other standard-model particles), assuming that it is the lightest superparticle in the minimal-supersymmetricstandard-model (MSSM) sector. Then, cosmologically, the lightest neutralino becomes an important source of the superWIMP, which is now a candidate of cold dark matter. The important point is that the lifetime of the lightest neutralino is very long; in the thermal history of the universe, the decay occurs after the lightest neutralino freezes out from the thermal bath. In this case, abundance of the superWIMP produced by the decay of the lightest neutralino is given by the freeze-out density of the lightest neutralino. Thus, even if one of the superWIMPs is the LSP, precise determination of the freeze-out density of the lightest neutralino is also important. $\# 2$

This paper is organized as follows. In the next section, we summarize the framework of our study. Then, in Section 3, we present formulas for QCD correction to the process $\chi_{1}^{0} \chi_{1}^{0} \rightarrow q \bar{q}(g)$. In Section 4, we calculate the relic density of the lightest neutralino and discuss implications of the QCD correction to the calculation of the dark matter density. Section 5 is devoted to conclusions and discussion.

\section{Framework}

In our study, we work in the framework of the MSSM. In addition, we assume that the lightest neutralino $\chi_{1}^{0}$ becomes the LSP and hence is stable. In general, the mass and mixing parameters of superparticles depend on various MSSM parameters. To make our points clearer, we adopt several simplifications.

In calculating $\Omega_{\chi_{1}^{0}}$, we need to fix properties of the lightest neutralino $\chi_{1}^{0}$, which are

\footnotetext{
${ }^{\# 2}$ One of the authors resisted glorifying supersymmetric models as scenarios for new physics, but the other authors ganged up and forced through the present style of Introduction.
} 
determined from the neutralino mass matrix $\mathcal{M}_{\chi^{0}}$. Denoting the $U(1)_{Y}$ and $S U(2)_{L}$ gaugino mass parameters as $m_{\mathrm{G} 1}$ and $m_{\mathrm{G} 2}$ and supersymmetric Higgs mass as $\mu_{H}, \mathcal{M}_{\chi^{0}}$ is given by

$$
\mathcal{M}_{\chi^{0}}=\left(\begin{array}{cccc}
-m_{\mathrm{G} 1} & 0 & -g_{1} v \cos \beta & g_{1} v \sin \beta \\
0 & -m_{\mathrm{G} 2} & g_{2} v \cos \beta & -g_{2} v \sin \beta \\
-g_{1} v \cos \beta & g_{2} v \cos \beta & 0 & \mu_{H} \\
g_{1} v \sin \beta & -g_{2} v \sin \beta & \mu_{H} & 0
\end{array}\right),
$$

where $g_{1}$ and $g_{2}$ are gauge coupling constants for for $U(1)_{Y}$ and $S U(2)_{L}$ gauge groups, respectively, $v \simeq 174 \mathrm{GeV}$ is the (total) vacuum expectation value of the Higgs boson, and $\tan \beta$ is the ratio of the vacuum expectation values of the up- and down-type Higgs bosons. This mass matrix is diagonalized by a unitary matrix $U_{\chi^{0}}$ as

$$
U_{\chi^{0}}^{T} \mathcal{M}_{\chi^{0}} U_{\chi^{0}}=\operatorname{diag}\left(m_{\chi_{1}^{0}}, m_{\chi_{2}^{0}}, m_{\chi_{3}^{0}}, m_{\chi_{4}^{0}}\right),
$$

and the lightest neutralino is given by

$$
\chi_{1}^{0}=\left[U_{\chi^{0}}\right]_{11}^{*} \tilde{B}+\left[U_{\chi^{0}}\right]_{21}^{*} \tilde{W}^{0}+\left[U_{\chi^{0}}\right]_{31}^{*} \tilde{H}_{d}^{0}+\left[U_{\chi^{0}}\right]_{41}^{*} \tilde{H}_{u}^{0},
$$

where $\tilde{B}, \tilde{W}^{0}, \tilde{H}_{u}^{0}$, and $\tilde{H}_{d}^{0}$ are Bino, (neutral) Wino, up-type Higgsino, and down-type Higgsino, respectively.

In our study, we adopt the grand-unified-theory (GUT) relation among gaugino masses. In this case we obtain the relation between $U(1)_{Y}$ and $S U(2)_{L}$ gaugino masses, $m_{\mathrm{G} 1}$ and $m_{\mathrm{G} 2}$, as

$$
\frac{m_{\mathrm{G} 2}}{g_{2}^{2}}=\frac{3}{5} \frac{m_{\mathrm{G} 1}}{g_{1}^{2}}
$$

Then, since $m_{\mathrm{G} 2}>m_{\mathrm{G} 1}$, the lightest neutralino is approximately given by a linear combination of Bino and Higgsinos. We will see that the QCD correction to the process $\chi_{1}^{0} \chi_{1}^{0} \rightarrow t \bar{t}(g)$ becomes most significant when the lightest neutralino is mostly Bino-like but is with a sizable Higgsino contamination. Notice that the formulas for the QCD correction which will be given in the next section are independent of the relation among the gauginos.

With the unitary matrix $U_{\chi^{0}}$, the $Z-\chi_{1}^{0}-\chi_{1}^{0}$ vertex, which is relevant for the process $\chi_{1}^{0} \chi_{1}^{0} \rightarrow$ $q \bar{q}(g)$, is given in the form

$$
\mathcal{L}_{Z \chi^{0} \chi^{0}}=\frac{1}{2} A_{\chi} \bar{\chi}_{1}^{0} \gamma_{\mu} \gamma_{5} \chi_{1}^{0} Z_{\mu}
$$

where

$$
A_{\chi}=\frac{1}{2} g_{z}\left(\left[U_{\chi^{0}}\right]_{31}^{*}\left[U_{\chi^{0}}\right]_{31}-\left[U_{\chi^{0}}\right]_{41}^{*}\left[U_{\chi^{0}}\right]_{41}\right),
$$

with $g_{Z} \equiv \sqrt{g_{1}^{2}+g_{2}^{2}}$. When $m_{\mathrm{G} 1} \ll \mu_{H},\left[U_{\chi^{0}}\right]_{11} \simeq 1$ while $\left[U_{\chi^{0}}\right]_{i 1}(i=2,3,4)$ become close to 0 . In this case, the lightest neutralino is almost Bino and its interactions with 
gauge and Higgs bosons are suppressed. Consequently, dominant annihilation processes are into lepton pairs (as far as the sleptons are lighter than the squarks) and hence are $p$-wave processes. When $\mu_{H}$ is relatively small, on the contrary, Higgsino component in the lightest neutralino becomes enhanced and $\left[U_{\chi^{0}}\right]_{31}$ and $\left[U_{\chi^{0}}\right]_{41}$ become sizable. In this case, the lightest neutralino pair dominantly annihilates into $t \bar{t}, W^{+} W^{-}$, and $Z Z$ final states since they are $s$-wave processes.

We also present the interaction of the neutralino with the lightest (standard-model-like) Higgs boson $h$. The interaction term is given in the form

$$
\mathcal{L}_{h \chi^{0} \chi^{0}}=\frac{1}{2} Y_{\chi} \bar{\chi}_{1}^{0} \chi_{1}^{0} h
$$

In our following study, we consider the so-called decoupling limit where all the physical Higgs bosons except the standard-model-like Higgs $h$ are very heavy. In this case, we can neglect effects of heavier Higgses on the annihilation processes of the lightest neutralino. In addition, in the decoupling limit, $Y_{\chi}$ is well approximated by $\# 3$

$$
Y_{\chi}=-\left(g_{2}\left[U_{\chi^{0}}\right]_{21}-g_{1}\left[U_{\chi^{0}}\right]_{11}\right)\left(\left[U_{\chi^{0}}\right]_{31} \cos \beta-\left[U_{\chi^{0}}\right]_{41} \sin \beta\right) .
$$

(Here and hereafter, we work in the bases where the matrix $U_{\chi^{0}}$ is real.)

In the calculation of $\Omega_{\chi_{1}^{0}}$, sfermions may also contribute. In the following, we pay particular attention to the case where the annihilation process of $\chi_{1}^{0}$ is dominated by $s$-wave processes. In such a case, sfermion-exchange diagrams are mostly subdominant since they induce $p$-wave processes. One exception may be $t$-channel exchange of stops. We assume that the stops are heavy and their contribution to the pair annihilation of $\chi_{1}^{0}$ is negligible. In addition, we also assume that heavier Higgs bosons are heavy enough so that they do not significantly affect $\Omega_{\chi_{1}^{0}}$. Such a mass spectrum with heavy scalars is realized in, for example, focus-point models [5].

\section{QCD Correction to $\sigma_{\chi_{1}^{0} \chi_{1}^{0} \rightarrow q \bar{q}(g)}$}

We calculate the NLO QCD correction to the cross section for neutralino pair annihilation into $q \bar{q}(g)$ final state. In this paper, we consider the case where the squarks (in particular, stops) are much heavier than the $Z$-boson. This is the case in a large class of supersymmetric models, partially due to renormalization group effects via gluino mass. In such models, the decays $\chi_{1}^{0} \chi_{1}^{0} \rightarrow q \bar{q}(g)$ are induced dominantly via $Z$ and $h$-boson exchange diagrams. When the initial-state neutralinos are non-relativistic, $Z$-boson exchange diagram turns out to be most important. $Z$ and $h$-boson exchange contributions do not interfere, since the $P$ (parity) properties of $Z$ and $h$ are different; hence we may discuss their contributions separately.

\footnotetext{
${ }^{\# 3}$ In the general case, $Y_{\chi}$ is given by replacing $\beta \rightarrow \alpha+\frac{1}{2} \pi$, where $\alpha$ is the mixing angle in the CP-even Higgs sector. (See, for example, [12].)
} 


\section{1 $O\left(\alpha_{s}\right)$ correction}

We first consider $O\left(\alpha_{s}\right)$ contributions from the one-loop and real-gluon emission processes. As will be discussed below, QCD corrections are enhanced when the final-state quarks are non-relativistic due to boundstate effects. In such a case, it is necessary to take account of (some part of) higher-order corrections in order to obtain reliable predictions. This will be treated in the next subsection.

We start with the contribution of the $Z$-boson exchange diagrams. The relevant interaction of the $Z$-boson with quarks can be parameterized as

$$
\mathcal{L}_{Z q \bar{q}}=\sum_{q} \bar{q} \gamma_{\mu}\left(V_{q}+A_{q} \gamma_{5}\right) q Z_{\mu}
$$

As we will see, in the calculation of the relic density of $\chi_{1}^{0}, t \bar{t}(g)$ final state is of particular interest. For the top quark,

$$
V_{t}=\frac{1}{2 g_{Z}}\left(\frac{1}{2} g_{2}^{2}-\frac{5}{6} g_{1}^{2}\right), \quad A_{t}=-\frac{1}{4} g_{Z} .
$$

We should consider two contributions simultaneously; one is the virtual correction to $Z$ $q-\bar{q}$ vertex and the other is the real gluon emission process $\chi_{1}^{0} \chi_{1}^{0} \rightarrow Z^{*} \rightarrow q \bar{q} g$. Both of them, individually, are infrared divergent, but these divergences cancel when both contributions are summed. Let us denote the Feynman amplitude for the process $\chi_{1}^{0} \chi_{1}^{0} \rightarrow Z^{*} \rightarrow q \bar{q}(g)$ as

$$
\mathcal{M}_{\chi_{1}^{0} \chi_{1}^{0} \rightarrow Z^{*} \rightarrow q \bar{q}(g)}=\Lambda_{\mu}^{(\chi)} D_{\mu \nu}^{(Z)} \Lambda_{\nu}^{(q)} .
$$

Here, $D_{\mu \nu}^{(Z)}=\frac{1}{Q^{2}-m_{Z}^{2}}\left(g_{\mu \nu}-\frac{Q_{\mu} Q_{\nu}}{m_{Z}^{2}}\right)$ is the propagator of $Z$-boson (with $Q_{\mu}$ being the total fourmomentum of the system), while $\Lambda_{\mu}^{(\chi)}$ and $\Lambda_{\nu}^{(q)}$ are bi-spinors (including coupling constants) consisting of the wave functions of the neutralino and quark, respectively. It is convenient to define the following quantity:

$$
\Pi_{\mu \nu}^{(q)}=\sum_{\text {spin }} \int d \Phi_{q \bar{q}}\left[\Lambda_{\mu}^{(q)} \Lambda_{\nu}^{(q) *}\right]_{\chi_{1}^{0} \chi_{1}^{0} \rightarrow Z^{*} \rightarrow q \bar{q}}+\sum_{\text {spin }} \int d \Phi_{q \bar{q} g}\left[\Lambda_{\mu}^{(q)} \Lambda_{\nu}^{(q) *}\right]_{\chi_{1}^{0} \chi_{1}^{0} \rightarrow Z^{*} \rightarrow q \bar{q} g},
$$

where the first and second terms represent contributions from the processes $\chi_{1}^{0} \chi_{1}^{0} \rightarrow Z^{*} \rightarrow$ $q \bar{q}$ (i.e., leading order + virtual correction) and $\chi_{1}^{0} \chi_{1}^{0} \rightarrow Z^{*} \rightarrow q \bar{q} g$ (i.e., real emission), respectively. In the above formula, the integrals $\int d \Phi$ are carried out over the final-state phase space, and the spin sums of the final-state particles are taken. The first and second terms in Eq. (3.4) individually contain infrared divergences, while $\Pi_{\mu \nu}^{(q)}$ is finite. We decompose $\Pi_{\mu \nu}^{(q)}$ into the vector and scalar parts as

$$
\Pi_{\mu \nu}^{(q)}=\Pi_{\mathrm{V}}^{(q)}\left(Q^{2}\right)\left(-g_{\mu \nu}+\frac{Q_{\mu} Q_{\nu}}{Q^{2}}\right)+\Pi_{\mathrm{S}}^{(q)}\left(Q^{2}\right) \frac{Q_{\mu} Q_{\nu}}{Q^{2}} .
$$




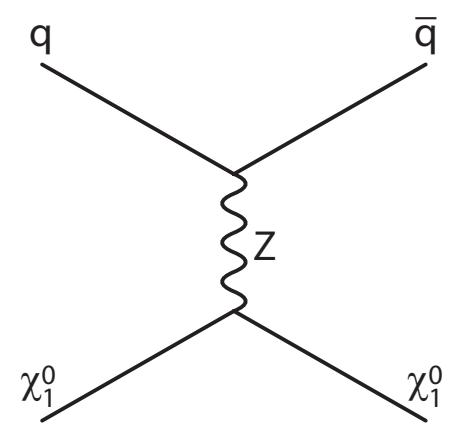

(a)

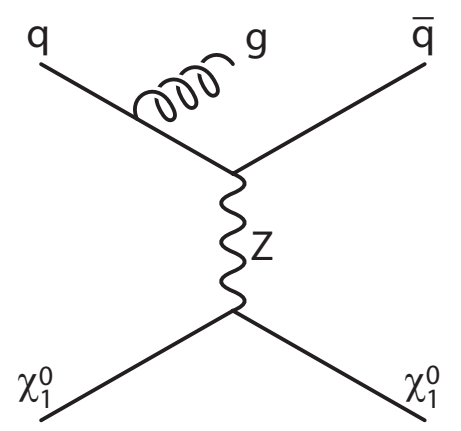

(c)

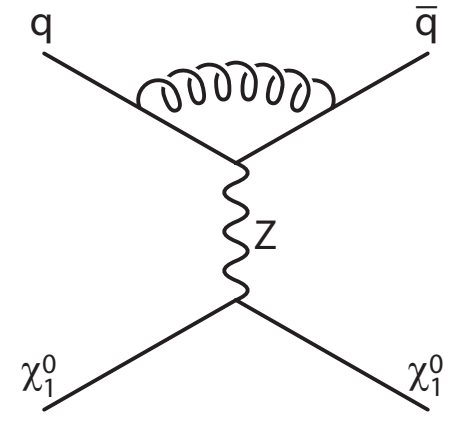

(b)

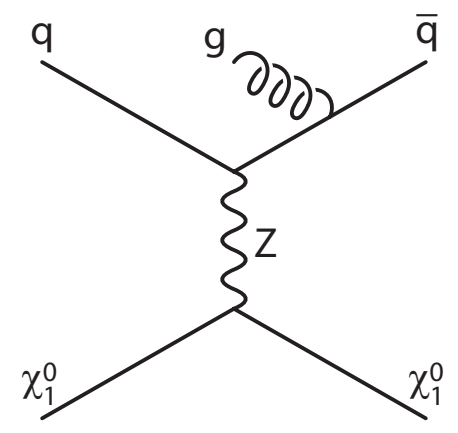

(d)

Figure 1: Feynman diagrams for the processes $\chi_{1}^{0} \chi_{1}^{0} \rightarrow Z^{*} \rightarrow q \bar{q}\left((\mathrm{a})\right.$ and (b)) and $\chi_{1}^{0} \chi_{1}^{0} \rightarrow$ $Z^{*} \rightarrow q \bar{q} g((\mathrm{c})$ and $(\mathrm{d}))$.

For the purpose of the calculation of the relic density of neutralino, we define ${ }^{\# 4}$

$$
\begin{aligned}
\mathcal{S}_{\chi_{1}^{0} \chi_{1}^{0} \rightarrow Z^{*} \rightarrow q \bar{q}(g)}\left(Q^{2}\right) & =2 v_{\chi_{1}^{0}} \sigma_{\chi_{1}^{0} \chi_{1}^{0} \rightarrow Z^{*} \rightarrow q \bar{q}(g)} \\
& =\frac{1}{4 Q^{2}}\left[\sum_{\text {spin }} \int d \Phi_{q \bar{q}}\left|\mathcal{M}_{\chi_{1}^{0} \chi_{1}^{0} \rightarrow Z^{*} \rightarrow q \bar{q}}\right|^{2}+\sum_{\text {spin }} \int d \Phi_{q \bar{q} g}\left|\mathcal{M}_{\chi_{1}^{0} \chi_{1}^{0} \rightarrow Z^{*} \rightarrow q \bar{q} g}\right|^{2}\right],
\end{aligned}
$$

where $v_{\chi_{1}^{0}}$ is the velocity of the lightest neutralino in the center-of-mass frame, which is given by

$$
v_{\chi_{1}^{0}}=\sqrt{1-\frac{4 m_{\chi_{1}^{0}}^{2}}{Q^{2}}} .
$$

\footnotetext{
\#4 It is often the case that the quantity $\mathcal{S}$ is expressed as $v_{\text {rel }} \sigma$, where $v_{\text {rel }}$ is the relative velocity of the initial-state neutralinos and $\sigma$ is the neutralino pair-annihilation cross section.
} 
In addition, $\sigma_{\chi_{1}^{0} \chi^{0} \rightarrow Z^{*} \rightarrow q \bar{q}(g)}$ is the total cross section for the process $\chi_{1}^{0} \chi^{0} \rightarrow Z^{*} \rightarrow q \bar{q}(g)$, hence, in the final formula, the spins of neutralinos are understood to be averaged. As we will see, the function $\mathcal{S}_{\chi_{1}^{0} \chi^{0} \rightarrow Z^{*} \rightarrow q \bar{q}(g)}$ plays an important role in the calculation of $\Omega_{\chi_{1}^{0}}$. With $\Pi_{\mathrm{V}}^{(q)}$ and $\Pi_{\mathrm{S}}^{(q)}, \mathcal{S}_{\chi_{1}^{0} \chi^{0} \rightarrow Z^{*} \rightarrow q \bar{q}(g)}$ is expressed as

$$
\mathcal{S}_{\chi_{1}^{0} \chi^{0} \rightarrow Z^{*} \rightarrow q \bar{q}(g)}\left(Q^{2}\right)=\frac{1}{Q^{2}}\left[\frac{Q^{2}-4 m_{\chi_{1}^{0}}^{2}}{\left(Q^{2}-m_{Z}^{2}\right)^{2}} \Pi_{\mathrm{V}}^{(q)}\left(Q^{2}\right)+\frac{2 m_{\chi_{1}^{0}}^{2}}{m_{Z}^{4}} \Pi_{\mathrm{S}}^{(q)}\left(Q^{2}\right)\right]\left|A_{\chi}\right|^{2} .
$$

Importantly, the first term in the parenthesis is proportional to $Q^{2}-4 m_{\chi_{1}^{0}}^{2}$; as a result, it is suppressed when the initial state neutralinos are non-relativistic. Thus, in such a case, the contribution of $\Pi_{\mathrm{V}}^{(q)}$ becomes negligible. In addition, $\Pi_{\mathrm{S}}^{(q)}$ is proportional to the masssquared of the final-state quark. Since the relic density is determined by the cross section at $Q^{2} \sim 4 m_{\chi_{1}^{0}}^{2}$, we pay particular attention to the process $\chi_{1}^{0} \chi_{1}^{0} \rightarrow Z^{*} \rightarrow t \bar{t}(g)$ and neglect other processes concerning lighter quarks, in calculating the relic density of $\chi_{1}^{0}$.

In this subsection, we present the formulas for $\Pi_{\mathrm{V}}^{(q)}$ and $\Pi_{\mathrm{A}}^{(q)}$ up to $O\left(\alpha_{s}\right)$. Corresponding Feynman diagrams are shown in Fig. (1. At the tree level, we obtain $\# 5$

$$
\begin{aligned}
& {\left[\Pi_{\mathrm{V}}^{(q)}\left(Q^{2}\right)\right]_{\text {tree }}=\frac{m_{q}^{2}}{3 \pi\left(1-v_{q}^{2}\right)} v_{q}\left[\left(3-v_{q}^{2}\right)\left|V_{q}\right|^{2}+2 v_{q}^{2}\left|A_{q}\right|^{2}\right],} \\
& {\left[\Pi_{\mathrm{S}}^{(q)}\left(Q^{2}\right)\right]_{\text {tree }}=\frac{m_{q}^{2}}{\pi} v_{q}\left|A_{q}\right|^{2},}
\end{aligned}
$$

where

$$
v_{q}=\sqrt{1-\frac{4 m_{q}^{2}}{Q^{2}}}
$$

All information on the QCD correction (at $O\left(\alpha_{s}\right)$ ) is contained in $\Pi_{\mathrm{V}}^{(q)}$ and $\Pi_{\mathrm{S}}^{(q)}$. In the calculation of the virtual contribution (Fig. [1(b)), we adopt the on-mass-shell renormalization condition to determine the vertex counter term: we require that the $O\left(\alpha_{s}\right)$ correction to the vector vertex (which is proportional to $V_{q}$ ) vanishes when $Q^{2} \rightarrow 0$. Then, $O\left(\alpha_{s}\right)$ correction to $\Pi_{\mathrm{V}}^{(q)}$ is found to be [13]

$$
\begin{aligned}
{\left[\Pi_{\mathrm{V}}^{(q)}\left(Q^{2}\right)\right]_{O\left(\alpha_{s}\right)}=} & \frac{\alpha_{s} C_{2} m_{q}^{2}}{24 \pi^{2}\left(1-v_{q}^{2}\right)}\left|V_{q}\right|^{2}\left[8\left(3-v_{q}^{2}\right) A\left(v_{q}\right)+6 v_{q}\left(5-3 v_{q}^{2}\right)\right. \\
& \left.+\left(33+22 v_{q}^{2}-7 v_{q}^{4}\right) \ln \frac{1+v_{q}}{1-v_{q}}\right] \\
& +\frac{\alpha_{s} C_{2} m_{q}^{2}}{48 \pi^{2}\left(1-v_{q}^{2}\right)}\left|A_{q}\right|^{2}\left[32 v_{q}^{2} A\left(v_{q}\right)+6 v_{q}\left(-7+10 v_{q}^{2}+v_{q}^{4}\right)\right. \\
& \left.+\left(21+59 v_{q}^{2}+19 v_{q}^{4}-3 v_{q}^{6}\right) \ln \frac{1+v_{q}}{1-v_{q}}\right]
\end{aligned}
$$

${ }^{\# 5}$ At $O\left(\alpha_{s}\right)$, the correction from real gluon emission is also from tree diagrams. $\left[\Pi_{\mathrm{V}}^{(q)}\right]_{\text {tree }}$ and $\left[\Pi_{\mathrm{S}}^{(q)}\right]_{\text {tree }}$ do not include such contributions. 
where $C_{2}=\frac{4}{3}$ is quadratic Casimir operator for the fundamental representation of $S U(3)$,

$$
\begin{aligned}
A\left(v_{q}\right)= & \left(1+v_{q}^{2}\right)\left[4 \mathrm{Li}_{2}\left(\frac{1-v_{q}}{1+v_{q}}\right)+2 \mathrm{Li}_{2}\left(-\frac{1-v_{q}}{1+v_{q}}\right)-3 \ln \frac{2}{1+v_{q}} \ln \frac{1+v_{q}}{1-v_{q}}\right. \\
& \left.-2 \ln v_{q} \ln \frac{1+v_{q}}{1-v_{q}}\right]-3 v_{q} \ln \frac{4}{1-v_{q}^{2}}-4 v_{q} \ln v_{q},
\end{aligned}
$$

and $\operatorname{Li}_{2}(x)$ is the dilogarithm (Spence) function

$$
\operatorname{Li}_{2}(x)=-\int_{0}^{x} d t \frac{\ln (1-t)}{t} .
$$

In addition, $O\left(\alpha_{s}\right)$ correction to $\Pi_{\mathrm{S}}^{(q)}$ is given by

$$
\left[\Pi_{\mathrm{S}}^{(q)}\left(Q^{2}\right)\right]_{O\left(\alpha_{s}\right)}=\frac{\alpha_{s} C_{2} m_{q}^{2}}{16 \pi^{2}}\left|A_{q}\right|^{2}\left[16 A\left(v_{q}\right)+6 v_{q}\left(7-v_{q}^{2}\right)+\left(19+2 v_{q}^{2}+3 v_{q}^{4}\right) \ln \frac{1+v_{q}}{1-v_{q}}\right] .
$$

We have checked that the ratio $\left[\Pi_{\mathrm{S}}^{(q)}\left(Q^{2}\right)\right]_{O\left(\alpha_{s}\right)} /\left[\Pi_{\mathrm{S}}^{(q)}\left(Q^{2}\right)\right]_{\text {tree }}$ coincides with the correction factor for the decay rate of the pseudo-scalar Higgs boson given in [14].

We can also calculate the $h$-exchange contributions. Although the process $\chi_{1}^{0} \chi_{1}^{0} \rightarrow h^{*} \rightarrow$ $t \bar{t}(g)$ is $p$-wave suppressed and hence is subdominant, we write down the formula for the QCD correction to this process to make this paper self-contained. Let us denote the $h q \bar{q}$ vertex as

$$
\mathcal{L}_{h q \bar{q}}=\sum_{q} Y_{q} \bar{q} q h
$$

and define

$$
\mathcal{S}_{\chi_{1}^{0} \chi_{1}^{0} \rightarrow h^{*} \rightarrow q \bar{q}(g)}\left(Q^{2}\right)=2 v_{\chi_{1}^{0}} \sigma_{\chi_{1}^{0} \chi_{1}^{0} \rightarrow h^{*} \rightarrow q \bar{q}(g)} .
$$

Adopting on-mass-shell renormalization condition for the $h-q-\bar{q}$ vertex, the coupling constant $Y_{q}$ is related to the (on-shell) mass of $q$ as

$$
Y_{q}=\frac{m_{q}}{\sqrt{2} v}
$$

Then, $\mathcal{S}_{\chi_{1}^{0} \chi_{1}^{0} \rightarrow h^{*} \rightarrow q \bar{q}(g)}$ is given by

$$
\mathcal{S}_{\chi_{1}^{0} \chi_{1}^{0} \rightarrow h^{*} \rightarrow q \bar{q}(g)}\left(Q^{2}\right)=\frac{m_{q}^{2}\left(Q^{2}-4 m_{\chi_{1}^{0}}^{2}\right)}{8 \pi Q^{2}\left(Q^{2}-m_{h}^{2}\right)^{2}} v_{q}^{3}\left|Y_{q} Y_{\chi}\right|^{2}\left(1+\frac{C_{2} \alpha_{s}}{\pi} \Delta_{h}\right),
$$

where 14

$$
\Delta_{h}=\frac{1}{v_{q}} A\left(v_{q}\right)+\frac{1}{16 v_{q}^{3}}\left(3+34 v_{q}^{2}-13 v_{q}^{4}\right) \ln \frac{1+v_{q}}{1-v_{q}}+\frac{3}{8 v_{q}^{2}}\left(-1+7 v_{q}^{2}\right) .
$$


For the calculation of the relic density of neutralino, the $s$-wave contribution plays the most significant role. As we mentioned, for the $s$-wave process, $t \bar{t}(g)$ final state is most important while the effects of lighter quarks are unimportant (if we restrict ourselves to the $Z$-exchange diagrams). Thus, in order to see the size of the QCD correction to the $s$-wave part, we define

$$
R_{O\left(\alpha_{s}\right)}\left(Q^{2}\right)=\frac{\left[\Pi_{\mathrm{S}}^{(t)}\left(Q^{2}\right)\right]_{O\left(\alpha_{s}\right)}}{\left[\Pi_{\mathrm{S}}^{(t)}\left(Q^{2}\right)\right]_{\text {tree }}} .
$$

We can see that the $O\left(\alpha_{s}\right)$ correction to the $s$-wave part is enhanced when $v_{t}$ becomes smaller. Indeed, expanding $R_{O\left(\alpha_{s}\right)}$ around $v_{t}=0$, we obtain

$$
R_{O\left(\alpha_{s}\right)}=\frac{C_{2} \alpha_{s}}{\pi}\left[\frac{\pi^{2}}{2 v_{t}}-3+O\left(v_{t}\right)\right] .
$$

This ratio is inversely proportional to $v_{t}$ when $v_{t} \ll 1$. As a result, the QCD correction largely enhances the neutralino annihilation cross section especially when the final-state top and anti-top quarks are non-relativistic. Thus, in the calculation of the relic density of the lightest neutralino, QCD correction may become important.

In fact, it is well known that QCD corrections are enhanced as $v_{t}$ becomes smaller, due to boundstate effects. Naive perturbative expansion breaks down in the region $v_{t} \lesssim \alpha_{s}$. In this region, systematic treatment of boundstate effects are known for heavy quarks, such as top quark. Up to NLO, this corresponds (formally) to the resummation of terms of the form $\left(\alpha_{s} / v_{t}\right)^{k}$ and $v_{t}\left(\alpha_{s} / v_{t}\right)^{k}=\alpha_{s}\left(\alpha_{s} / v_{t}\right)^{k-1}$ for $0 \leq k<\infty$ (where $k$ is an integer). In the next subsection, we compute the QCD correction reliably by resumming all these corrections.

\subsection{NLO annihilation cross section close to $q \bar{q}$ threshold}

We compute the QCD correction to the process $\chi_{1}^{0} \chi_{1}^{0} \rightarrow q \bar{q}(g)$ close to $q \bar{q}$ threshold up to NLO (including boundstate effects). Our main concern is to calculate the relic density of the lightest neutralino; for this purpose, the $s$-wave part of the cross section is most important. Thus, in this subsection, we concentrate on the QCD correction to the $s$-wave part.

Following the standard framework developed for the threshold cross sections, for example, for the process $e^{+} e^{-} \rightarrow t \bar{t}[8$, the NLO annihilation cross section (when the final-state quarks are non-relativistic) is given as follows:

$$
\left[\Pi_{\mathrm{S}}^{(q)}\left(Q^{2}\right)\right]_{\mathrm{NLO}, \mathrm{NR}}=F\left(Q^{2}\right)\left(1+\frac{C_{2} \alpha_{s}}{\pi} \Delta_{\mathrm{S}}^{(\mathrm{hard})}\right)\left[\Pi_{\mathrm{S}}^{(q)}\left(Q^{2}\right)\right]_{\mathrm{tree}},
$$

where $\Delta_{\mathrm{S}}^{(\text {hard })}$ represents the hard-vertex correction given by

$$
\Delta_{\mathrm{S}}^{(\text {hard })}=\lim _{v_{q} \rightarrow 0}\left[\left(\frac{C_{2} \alpha_{s}}{\pi}\right)^{-1} \frac{\left[\Pi_{\mathrm{S}}^{(q)}\left(Q^{2}\right)\right]_{O\left(\alpha_{s}\right)}}{\left[\Pi_{\mathrm{S}}^{(q)}\left(Q^{2}\right)\right]_{\text {tree }}}-\frac{\pi^{2}}{2 v_{q}}\right]=-3 .
$$


Boundstate effects are included in $F\left(Q^{2}\right)$, which is given in terms of the Green functions of the non-relativistic Schrödinger equation:

$$
F\left(Q^{2}\right)=\frac{\operatorname{Im}[G(\overrightarrow{0} ; E)]}{\operatorname{Im}\left[G_{0}(\overrightarrow{0} ; E)\right]} .
$$

$G(\vec{x} ; E)$ is defined by

$$
\left[\left(E+i \Gamma_{q}\right)-\left\{-\frac{\nabla^{2}}{m_{q}}+V_{\mathrm{QCD}}^{(\mathrm{NLO})}\left(r ; \mu_{\mathrm{Bohr}}\right)\right\}\right] G(\vec{x} ; E)=\delta^{3}(\vec{x}) .
$$

Here, $V_{\mathrm{QCD}}^{(\mathrm{NLO})}\left(r ; \mu_{\mathrm{Bohr}}\right)$ denotes the static QCD potential up to NLO [15]; $E=\sqrt{Q^{2}}-2 m_{q}$ is the center-of-mass energy measured from the $q \bar{q}$ threshold, and $\Gamma_{q}$ is the total decay width of $q$. On the other hand, $G_{0}(\vec{x} ; E)$ is the non-relativistic Green function of a free $q \bar{q}$ pair, which is defined via Eq. (3.26) after setting $V_{\mathrm{QCD}}^{(\mathrm{NLO})}(r)$ to 0.

In the case of QED (i.e., when $V_{\mathrm{QCD}}(r)$ is replaced by the Coulomb potential $-\alpha_{\mathrm{QED}} / r$, with $\alpha_{\mathrm{QED}}$ being the fine-structure constant), $F\left(Q^{2}\right)$ reduces to the well-known Sommerfeld factor:

$$
\left[F\left(Q^{2}\right)\right]_{\mathrm{QED}}=\frac{z}{1-e^{-z}}
$$

where

$$
z=\frac{\pi \alpha_{\mathrm{QED}}}{v_{q}}
$$

In this case, it is easy to see via Eqs. (3.22) ${ }^{\# 6}$ and (3.27) that the QED correction including the boundstate effects is twice as large as the (naive) one-loop correction at $\sqrt{Q^{2}}=2 m_{q}$. One may regard this as a reference for the significance of boundstate effects, with respect to the naive one-loop correction, in the limit where the running of the strong coupling constant may be neglected (since it corresponds to the limit where the static QCD potential reduces to the Coulomb potential); qualitatively it is a reasonable approximation for final-state quarks as heavy as the top quark.

With Eq. (3.23), we also define the magnitude of the QCD correction relative to the LO contribution as

$$
R\left(Q^{2}\right)_{\mathrm{NLO}, \mathrm{NR}}=\frac{\left[\Pi_{\mathrm{S}}^{(q)}\left(Q^{2}\right)\right]_{\mathrm{NLO}, \mathrm{NR}}}{\left[\Pi_{\mathrm{S}}^{(q)}\left(Q^{2}\right)\right]_{\mathrm{tree}}}-1 .
$$

\subsection{QCD-corrected neutralino annihilation cross section}

In calculating the relic density of the lightest neutralino, the annihilation cross section for $Q^{2} \sim 4 m_{\chi_{1}^{0}}^{2}$ is important. This is because the lightest neutralinos decouple from the thermal bath when the cosmic temperature is much lower than $m_{\chi_{1}^{0}}$; at such low temperature, neutralinos are non-relativistic.

${ }^{\# 6}$ One should replace $C_{2} \alpha_{s}$ by $\alpha_{\mathrm{QED}}$. 
To see the importance of the QCD correction in such a case, in Fig. 2. we plot $R_{O\left(\alpha_{s}\right)}\left(Q^{2}=\right.$ $\left.4 m_{\chi_{1}^{0}}^{2}\right)$ and $R_{\mathrm{NLO}, \mathrm{NR}}\left(Q^{2}=4 m_{\chi_{1}^{0}}^{2}\right)$ as functions of $m_{\chi_{1}^{0}}$. It can be easily seen that the QCD correction significantly enhances the cross section. Since the tree-level cross section vanishes as $v_{t} \rightarrow 0$ while the QCD corrections stay constant, $R_{O\left(\alpha_{s}\right)}\left(Q^{2}=4 m_{\chi_{1}^{0}}^{2}\right)$ and $R_{\mathrm{NLO}, \mathrm{NR}}\left(Q^{2}=\right.$ $\left.4 m_{\chi_{1}^{0}}^{2}\right)$ diverge as $m_{\chi_{1}^{0}} \rightarrow m_{t}$. As a result, the size of the QCD correction becomes comparable to the tree-level contribution when the final-state top and anti-top quarks are non-relativistic. This fact has important implications to the calculation of $\Omega_{\chi_{1}^{0}}$ when the lightest neutralino dominantly annihilates into a $t \bar{t}$ pair.

Estimate of the annihilation cross section using the one-loop QCD correction $R_{O\left(\alpha_{s}\right)}$ is valid when $\alpha_{s} \ll v_{t}$ and $\alpha_{s} \ll 1$, since both (naive) higher-order contributions $\alpha_{s}^{n}$ and higherorder boundstate contributions $\left(\alpha_{s} / v_{t}\right)^{n}$ are suppressed. On the other hand, estimate of the QCD correction by $R_{\mathrm{NLO}, \mathrm{NR}}$ is valid when $v_{t}, \alpha_{s} \ll 1$. Thus, in the overlap region when $\alpha_{s} \ll v_{t} \ll 1$ hold, both $R_{O\left(\alpha_{s}\right)}$ and $R_{\mathrm{NLO}, \mathrm{NR}}$ are reasonable approximations. According to Fig. 2, when the lightest neutralino mass is in the range $200-220 \mathrm{GeV}, R_{O\left(\alpha_{s}\right)}\left(Q^{2}=\right.$ $\left.4 m_{\chi_{1}^{0}}^{2}\right)$ is reasonably close to $R_{\mathrm{NLO}, \mathrm{NR}}\left(Q^{2}=4 m_{\chi_{1}^{0}}^{2}\right.$ ) (with respect to the estimate of the NNLO correction). On the contrary, with smaller $m_{\chi_{1}^{0}}$, boundstate effects become important. Indeed, in such a case, $R_{O\left(\alpha_{s}\right)}\left(Q^{2}=4 m_{\chi_{1}^{0}}^{2}\right)$ and $R_{\mathrm{NLO}, \mathrm{NR}}\left(Q^{2}=4 m_{\chi_{1}^{0}}^{2}\right)$ differ significantly, and the latter prediction is more reliable.

Since the QCD correction enhances the cross section $\sigma_{\chi_{1}^{0} \chi_{1}^{0} \rightarrow t \bar{t}(g)}$, it may play an important role in the calculation of the relic density of $\chi_{1}^{0}$. In the next section, we will discuss how the relic density changes as we take account of the QCD correction.

\section{Relic Density of the Lightest Neutralino}

In this section, we calculate the relic density of the lightest neutralino. We include the QCD correction to the neutralino annihilation cross section given in the previous section and see its effects on the calculation of the relic density of the neutralino.

QCD corrections for the process $\chi_{1}^{0} \chi_{1}^{0} \rightarrow q \bar{q}(g)$ may be important for the precise calculation of $\Omega_{\chi_{1}^{0}}$. When the MSSM parameters will be precisely measured in future collider experiments after the discoveries of superparticles, cross sections of the pair annihilation processes of $\chi_{1}^{0}$ will be calculated in detail. Using the formulas given in the previous section, it is straightforward to include the QCD correction to the processes $\chi_{1}^{0} \chi_{1}^{0} \rightarrow q \bar{q}(g)$. Here, we show the importance of the QCD correction. To make our point clearer, here we take into account only the most important annihilation processes and neglect sub-dominant ones.

In order to calculate the relic density, we should follow the evolution of the number density of the lightest neutralino $n_{\chi_{1}^{0}}$, which is governed by the Boltzmann equation. Denoting the energy distribution function of the lightest neutralino as $f_{\chi_{1}^{0}}, \# 7$ the Boltzmann equation is

\footnotetext{
${ }^{\# 7} n_{\chi_{1}^{0}}$ and $f_{\chi_{1}^{0}}$ are related to each other by $n_{\chi_{1}^{0}}=\int \frac{d^{3} \vec{p}_{\chi_{1}^{0}}}{(2 \pi)^{3}} f_{\chi_{1}^{0}}\left(p_{\chi_{1}^{0}}\right)$.
} 


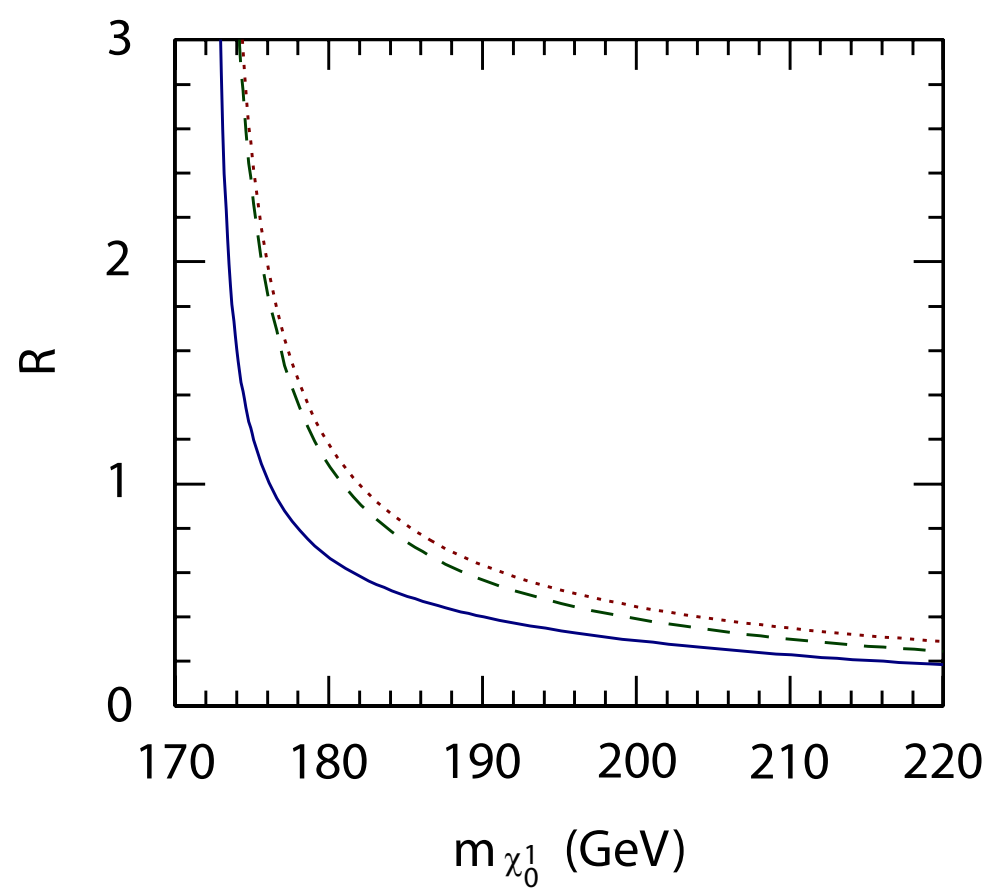

Figure 2: QCD correction to the cross section $\sigma_{\chi_{1}^{0} \chi_{1}^{0} \rightarrow q \bar{q}(g)}$ when the initial $\chi_{1}^{0}$ are at rest (i.e., $\left.Q^{2} \rightarrow 4 m_{\chi_{1}^{0}}^{2}\right)$ as a function of the LSP mass $m_{\chi_{1}^{0}}$. Three lines correspond to $R_{O\left(\alpha_{s}\right)}$ (solid), $R_{\mathrm{NLO}, \mathrm{NR}}$ with $\mu_{\mathrm{Bohr}}=20 \mathrm{GeV}$ (dashed), and $R_{\mathrm{NLO}, \mathrm{NR}}$ with $\mu_{\mathrm{Bohr}}=40 \mathrm{GeV}$ (dotted). The input values are taken as $\alpha_{s}\left(m_{t}\right)=0.1080$ (corresponding to $\alpha_{s}\left(m_{Z}\right)=0.1187$ ) and $m_{t}=172.5 \mathrm{GeV} . \mu_{\text {Bohr }}$ represents the renormalization scale in $V_{\mathrm{QCD}}^{(\mathrm{NLO})}(r)$, where a sensible choice of $\mu_{\text {Bohr }}$ is at the Bohr scale of $t \bar{t}$ resonances. We may regard the difference between the predictions for $\mu_{\mathrm{Bohr}}=20 \mathrm{GeV}$ and $40 \mathrm{GeV}$ as an estimate of contributions beyond NLO.

given by [16]

$$
\begin{aligned}
\frac{d n_{\chi_{1}^{0}}}{d t}+3 H n_{\chi_{1}^{0}}= & -\sum_{\text {processes }} \int d \Pi_{\chi_{1}^{0}} d \Pi_{\chi_{1}^{0}}^{\prime} d \Phi_{\mathrm{f}}\left|\mathcal{M}_{\chi_{1}^{0} \chi_{1}^{0} \rightarrow \mathrm{f}}\right|^{2} f_{\chi_{1}^{0}}\left(p_{\chi_{1}^{0}}\right) f_{\chi_{1}^{0}}\left(p_{\chi_{1}^{0}}^{\prime}\right) \\
& +(\text { production term }),
\end{aligned}
$$

where $H$ is the expansion rate of the universe. In the above formula, $\int d \Phi_{\mathrm{f}}$ is the integration over the phase space of final-state particles and we sum over all the relevant pair annihilation processes. In addition, the phase space integrations $\int d \Pi_{\chi_{1}^{0}}$ and $\int d \Pi_{\chi_{1}^{0}}^{\prime}$ are for the initial-state neutralinos (whose momenta are $p_{\chi_{1}^{0}}$ and $p_{\chi_{1}^{0}}^{\prime}$, respectively).

Even if the superparticles may not be in chemical equilibrium, they are expected to be in kinematical equilibrium since superparticles effectively interact with ordinary standardmodel particles in thermal bath. In addition, since the freeze out of the lightest neutralino occurs when $T \ll m_{\chi_{1}^{0}}$, we use the Boltzmann distribution function for neutralinos; at cosmic 
temperature $T$,

$$
f_{\chi_{1}^{0}}\left(p_{\chi_{1}^{0}}\right)=2 e^{-\left(p_{\chi_{1}^{0}, 0}-\mu_{\chi_{1}^{0}}\right) / T},
$$

where the factor of 2 represents the spin multiplicity of neutralino, $p_{\chi_{1}^{0}, 0}$ is the energy of $\chi_{1}^{0}$, and $\mu_{\chi_{1}^{0}}$ is the chemical potential.

In order to follow the evolution of $n_{\chi_{1}^{0}}$ for $T \ll m_{\chi_{1}^{0}}$, it is convenient to define

$$
x(T)=\frac{T}{m_{\chi_{1}^{0}}} .
$$

Once the temperature becomes lower than $m_{\chi_{1}^{0}}, x$ can be used as an expansion parameter; we expand various quantities using $x$. For example, the number density of the lightest neutralino is given by

$$
n_{\chi_{1}^{0}}=2 e^{-\left(m_{\chi_{1}^{0}}-\mu_{\chi_{1}^{0}}\right) / T}\left(\frac{m_{\chi_{1}^{0}} T}{2 \pi}\right)^{3 / 2}\left[1-\frac{15}{8} x+O\left(x^{2}\right)\right],
$$

where this formula is obtained by expanding the energy as

$$
p_{\chi_{1}^{0}, 0}=m_{\chi_{1}^{0}}+\frac{\left|\vec{p}_{\chi_{1}^{0}}\right|^{2}}{2 m_{\chi_{1}^{0}}}-\frac{\left|\vec{p}_{\chi_{1}^{0}}\right|^{4}}{8 m_{\chi_{1}^{0}}^{3}}+\cdots,
$$

with $\vec{p}_{\chi_{1}^{0}}$ being the three-momentum of $\chi_{1}^{0}$. Substituting Eq. (4.2) into Eq. (4.1), and applying the detailed balance theorem to determine the production term, the Boltzmann equation reduces to

$$
\frac{d n_{\chi_{1}^{0}}}{d t}+3 H n_{\chi_{1}^{0}}=-\left(1-\frac{3}{2} x\right) \mathcal{S}_{\text {tot }}\left(Q^{2}=4 m_{\chi_{1}^{0}}^{2}+6 x m_{\chi_{1}^{0}}^{2}\right)\left(n_{\chi_{1}^{0}}^{2}-n_{\chi_{1}^{0}}^{(\mathrm{eq}) 2}\right)+O\left(x^{2}\right),
$$

where $n_{\chi_{1}^{0}}^{(\mathrm{eq})}$ is the chemical-equilibrium value of the number density of $\chi_{1}^{0}$, which is obtained from Eq. (4.4) by taking $\mu_{\chi_{1}^{0}} \rightarrow 0$. In addition, $\mathcal{S}_{\text {tot }}$ is given by

$$
\mathcal{S}_{\text {tot }}=2 v_{\chi} \sigma_{\chi_{1}^{0} \chi_{1}^{0} \rightarrow \text { all }}
$$

with $\sigma_{\chi_{1}^{0} \chi_{1}^{0} \rightarrow \text { all }}$ being the total pair annihilation cross section of the lightest neutralino.\#8 Taking account of the correction of the order of $x, Q^{2}$ is shifted from $4 m_{\chi_{1}^{0}}^{2}$ to $4 m_{\chi_{1}^{0}}^{2}+6 x m_{\chi_{1}^{0}}^{2}$. This is due to the fact that, in the thermal bath, neutralinos have non-vanishing kinetic energy whose average is $\frac{3}{2} T$.

\footnotetext{
${ }^{\# 8}$ Here, we assume that the effects of coannihilation is negligible. For a precise calculation of the relic density, effects of the coannihilation should be taken into account [17. Once the relevant MSSM parameters are measured at the future collider experiments, however, effects of the coannihilation can be theoretically controlled. Thus, in our analysis, we do not include effects of the coannihilation, which are beyond our scope.
} 
From Eq. (4.5), several important features of $n_{\chi_{1}^{0}}$ are derived. When the cosmic temperature is high enough, production and annihilation rates of the lightest neutralino, which are $\sim \mathcal{S}_{\text {tot }} n_{\chi_{1}^{0}}$, are relatively high. At this epoch, scattering rate becomes much larger than the cosmic expansion rate $H$ and, consequently, the number density of $\chi_{1}^{0}$ keeps up with the equilibrium value $n_{\chi_{1}^{0}}^{(\mathrm{eq})}$. As the temperature decreases, however, the situation changes. Importantly, once the cosmic temperature becomes lower than $m_{\chi_{1}^{0}}, n_{\chi_{1}^{0}}^{(\mathrm{eq})}$ is Boltzmann suppressed. When $n_{\chi_{1}^{0}}$ (which is close to $n_{\chi_{1}^{0}}^{(\mathrm{eq})}$ at this epoch) becomes so suppressed that $\mathcal{S}_{\text {tot }} n_{\chi_{1}^{0}}$ becomes smaller than $H, \chi_{1}^{0}$ freezes out from the thermal bath. After the freeze out, the number of $\chi_{1}^{0}$ in comoving volume is conserved.

The freeze-out of the lightest neutralino occurs when $x^{-1} \sim 20$ and hence the lightest neutralinos are non-relativistic in thermal bath at the time of freeze out. Thus, the relic density is primarily determined by the behavior of the total cross section at $Q^{2} \rightarrow 4 m_{\chi_{1}^{0}}^{2}$, as can be understood from Eq. (4.5). When $A_{\chi}$ given in Eq. (2.6) is sizable, pair annihilation of the lightest neutralino is dominated by $s$-wave processes as $Q^{2} \rightarrow 4 m_{\chi_{1}^{0}}^{2}$. Thus, in such a case, $\Omega_{\chi_{1}^{0}}$ is mostly determined by the $s$-wave part of the total cross section. When all the sfermions and heavier Higgses are relatively heavy, the relevant $s$-wave processes are $\chi_{1}^{0} \chi_{1}^{0} \rightarrow t \bar{t}(g), W^{+} W^{-}$, and $Z Z$. In our analysis, we assume that this is the case and approximate

$$
\mathcal{S}_{\text {tot }} \simeq \mathcal{S}_{\chi_{1}^{0} \chi_{1}^{0} \rightarrow Z^{*} \rightarrow q \bar{q}(g)}+\mathcal{S}_{\chi_{1}^{0} \chi_{1}^{0} \rightarrow W^{+} W^{-}}+\mathcal{S}_{\chi_{1}^{0} \chi_{1}^{0} \rightarrow Z Z}
$$

Here, the second and third terms on the right-hand side represent the contributions from $W^{+} W^{-}$and $Z Z$ final states, respectively:

$$
\mathcal{S}_{\chi_{1}^{0} \chi_{1}^{0} \rightarrow W^{+} W^{-}, Z Z}=2 v_{\chi_{1}^{0} \sigma_{\chi_{1}^{0} \chi_{1}^{0} \rightarrow W^{+} W^{-}, Z Z}}
$$

with $\sigma_{\chi_{1}^{0} \chi_{1}^{0} \rightarrow W^{+} W^{-}, Z Z}$ being total cross section for the corresponding processes. We use the tree-level formulas for $\sigma_{\chi_{1}^{0} \chi_{1}^{0} \rightarrow W^{+} W^{-}}$and $\sigma_{\chi_{1}^{0} \chi_{1}^{0} \rightarrow Z Z}$. In addition, we evaluate these quantities at the threshold since their $Q^{2}$ dependences are weak when $x \ll 1$. (For $\chi_{1}^{0} \chi_{1}^{0} \rightarrow q \bar{q}(g), O(x)$ terms are considered unless otherwise mentioned.) Other processes, like $\chi_{1}^{0} \chi_{1}^{0} \rightarrow Z h$ and $h h$, are $p$-wave processes and are neglected in our study. If we include those processes, $\Omega_{\chi_{1}^{0}}$ may decrease by a few \% or so [17]; once the MSSM parameters will be experimentally determined, it will be straightforward to take account of effects of the $p$-wave processes. Furthermore, $\mathcal{S}_{\chi_{1}^{0} \chi_{1}^{0} \rightarrow q \bar{q}(g)}$ is calculated neglecting the $h$-exchange diagram since its contribution is $p$-wave suppressed.

We have numerically solved the Boltzmann equation (4.5) to determine the present value of $n_{\chi_{1}^{0}}$. We impose the initial condition $n_{\chi_{1}^{0}}=n_{\chi_{1}^{0}}^{(\mathrm{eq})}$ at the cosmic time where $\mathcal{S}_{\text {tot }} n_{\chi_{1}^{0}}^{(\mathrm{eq})} \gg H$ and follow the evolution of $n_{\chi_{1}^{0}}$. Then, we determine the number density of the lightest neutralino at $\mathcal{S}_{\text {tot }} n_{\chi_{1}^{0}}^{(\mathrm{eq})} \ll H$. In our following argument, it is convenient to define the yield variable

$$
Y_{\chi_{1}^{0}}=\frac{n_{\chi_{1}^{0}}}{s}
$$


with $s$ being the entropy density. Important property of this quantity is that $Y_{\chi_{1}^{0}}$ stays constant when $\mathcal{S}_{\text {tot }} n_{\chi_{1}^{0}}^{(\mathrm{eq})} \ll H$; indeed, we have numerically checked that the yield variable becomes a constant of time when the scattering rate is much smaller than the expansion rate. Using the yield variable after the freeze-out of $\chi_{1}^{0}$ (which we denote $Y_{\chi_{1}^{0}}^{(\text {now })}$ ), the density parameter is given by

$$
\Omega_{\chi_{1}^{0}}=m_{\chi_{1}^{0}} Y_{\chi_{1}^{0}}^{(\text {now })}\left[\frac{\rho_{\text {crit }}}{s^{(\text {now })}}\right]^{-1} .
$$

Here, $\rho_{\text {crit }}$ is the critical density and $s^{(\text {now })}$ is the present entropy density; numerically,

$$
\frac{\rho_{\text {crit }}}{s^{\text {(now) }}} \simeq 3.6 \times 10^{-9} h_{100}^{2} \mathrm{GeV} .
$$

In Fig. 3. we plot the density parameter of the lightest neutralino as a function of $m_{\chi_{1}^{0}}$. In the figure, we compare the results with and without the QCD correction to the process $\chi_{1}^{0} \chi_{1}^{0} \rightarrow t \bar{t}(g)$; we evaluate the cross section $\sigma_{\chi_{1}^{0} \chi_{1}^{0} \rightarrow t \bar{t}(g)}$ with NLO QCD correction with boundstate effects (solid), $O\left(\alpha_{s}\right)$ correction (dashed), and tree-level formula (dotted). In the calculation with the boundstate effects, we restrict ourselves to the case with $m_{\chi_{1}^{0}} \lesssim 210 \mathrm{GeV}$. This is because, when $m_{\chi_{1}^{0}}$ is too large, non-relativistic approximation used in the calculation becomes unreliable. Fortunately, however, as the lightest neutralino mass becomes larger than $\sim 200 \mathrm{GeV}$, result with the boundstate effects is in a very good agreement with that with $O\left(\alpha_{s}\right)$ contributions. This fact shows that QCD correction to the process $\chi_{1}^{0} \chi_{1}^{0} \rightarrow t \bar{t}(g)$ is well approximated by the one-loop formula when the lightest neutralino mass is larger than $\sim 200 \mathrm{GeV}$. On the contrary, when $m_{\chi_{1}^{0}}$ is smaller, difference between the one-loop result and the resummed one is quite large; in this case the latter prediction (solid) is more reliable.

We can see that the QCD correction significantly reduces the density parameter of the lightest neutralino when $m_{\chi_{1}^{0}}$ is close to the top-quark mass. Since the $t \bar{t}$ final state is more important than the gauge-boson final states when the lightest neutralino mass is relatively close to the top-quark mass, this fact can be easily understood from Fig. 2. As $m_{\chi_{1}^{0}}$ becomes larger, on the contrary, $\sigma_{\chi_{1}^{0} \chi_{1}^{0} \rightarrow W^{+} W^{-}, Z Z}$ becomes larger than $\sigma_{\chi_{1}^{0} \chi_{1}^{0} \rightarrow t \bar{t}(g)}$ and the gauge-boson final state becomes more important than the $t \bar{t}$ final state. In such a case, even if the QCD correction to the process $\chi_{1}^{0} \chi_{1}^{0} \rightarrow t \bar{t}(g)$ is sizable, $\Omega_{\chi_{1}^{0}}$ may not be affected so much. Indeed, we can see such a behavior in the figure. As the lightest neutralino mass becomes large enough, tree-level result becomes almost the same as the one with QCD correction although the QCD correction to $\sigma_{\chi_{1}^{0} \chi_{1}^{0} \rightarrow t \bar{t}(g)}$ is still more than $10 \%$.

In Fig. 3(a), we kept $O(x)$ terms of $\mathcal{S}_{\chi_{1}^{0} \chi_{1}^{0} \rightarrow t \bar{t}(g)}$. For comparison, we also calculated the density parameter with neglecting $x$-dependences (by taking $x \rightarrow 0$ ); the results are shown in Fig. 3(b). We can see that the resultant density parameters differ in two figures when the lightest neutralino mass becomes close to the top-quark mass. We have checked that the effects of $O(x)$ terms become larger as $m_{\chi_{1}^{0}}$ becomes closer to $m_{t}$.

In Figs. 4 and 5, we also plot the density parameter for the cases with $\mu_{H}=260 \mathrm{GeV}$ and $\mu_{H}=300 \mathrm{GeV}$, respectively. As we increase $\mu_{H}$, the WMAP value is realized with 


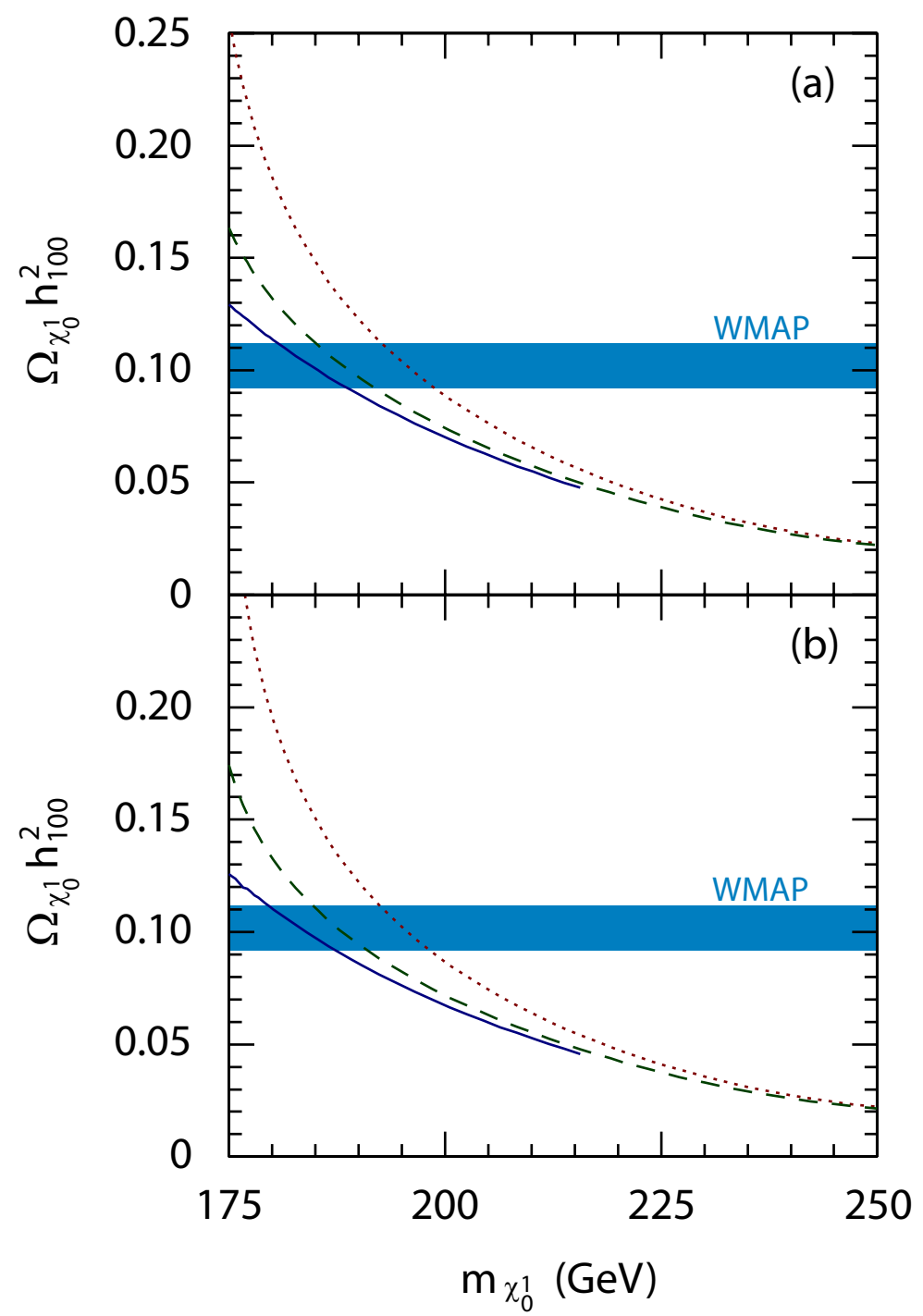

Figure 3: Density parameter of the lightest neutralino as a function of $m_{\chi_{1}^{0}}$. The cross section $\sigma_{\chi_{1}^{0} \chi_{1}^{0} \rightarrow t \bar{t}(g)}$ is evaluated with NLO QCD correction with boundstate effects (solid), $O\left(\alpha_{s}\right)$ correction (dashed), and tree-level formula (dotted). Here we take $\alpha_{s}\left(m_{Z}\right)=0.1189$, $m_{t}=172.5 \mathrm{GeV}$, and $\mu_{H}=280 \mathrm{GeV}$. The dark shaded band shows the dark matter density measured by the WMAP (see Eq. (1.1)). For (a) and (b), collision term for $t \bar{t}(g)$ final state is evaluated up to $O(x)$ and $O\left(x^{0}\right)$, respectively. 


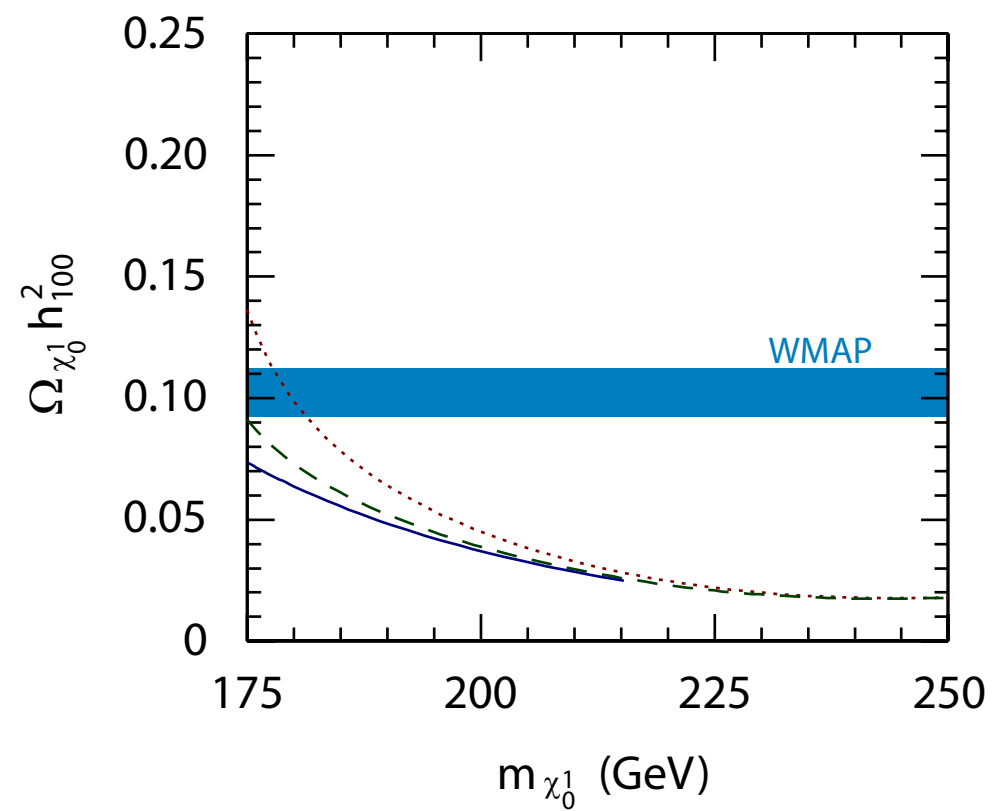

Figure 4: Same as Fig. 31(a) except for $\mu_{H}=260 \mathrm{GeV}$.

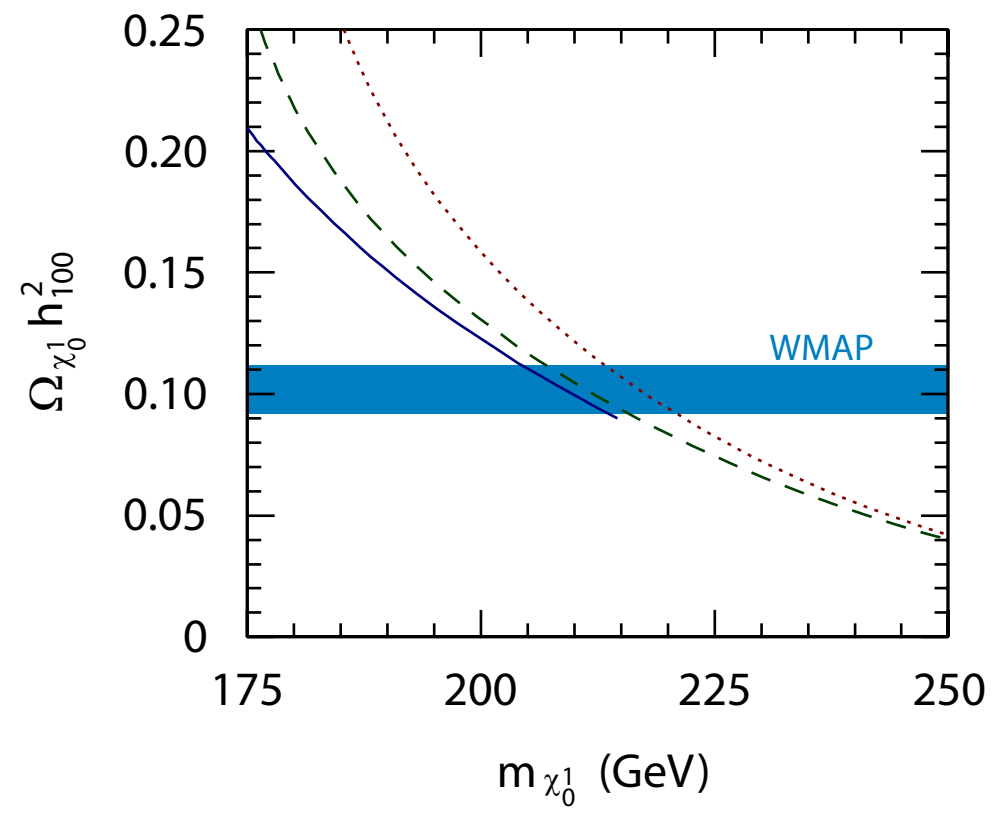

Figure 5: Same as Fig. 3(a) except for $\mu_{H}=300 \mathrm{GeV}$. 
larger value of $m_{\chi_{1}^{0}}$ due to the change of the mixing matrix of the neutralinos. These figures show that, with these values of $\mu_{H}$, the relic density of the lightest neutralino is significantly

affected when $m_{\chi_{1}^{0}}$ is close to $m_{t}$. As $\mu_{H}$ increases, however, QCD correction becomes less important in the parameter region where $\Omega_{\chi_{1}^{0}}$ agrees with the WMAP value.

\section{Conclusions and Discussion}

In this paper, we have calculated QCD correction to the process $\chi_{1}^{0} \chi_{1}^{0} \rightarrow q \bar{q}(g)$, and discussed its implications to the determination of $\Omega_{\chi_{1}^{0}}$. We have seen that the QCD correction enhances the cross section $\sigma_{\chi_{1}^{0} \chi_{1}^{0} \rightarrow q \bar{q}(g)}$ by $O(10 \%)$ when the final-state quarks are non-relativistic. Importantly, the lightest neutralino dominantly annihilates into a $t \bar{t}$ pair (as well as into $W^{+} W^{-}$and $Z Z$ ) when the lightest neutralino has sizable Higgsino components. In such a case, QCD correction may become large and significantly enhance the total annihilation cross section of the lightest neutralino. Since the QCD correction is enhanced close to the threshold by factors $\left(\alpha_{s} / v_{t}\right)^{k}$, the QCD correction becomes important in particular when $m_{\chi_{1}^{0}}$ is close to the top-quark mass. Since the relic density of the lightest neutralino is (approximately) inversely proportional to the total pair annihilation cross section, the relic density may be reduced by $O(10 \%)$ by the QCD correction. Thus, for a precise calculation of the relic density of the LSP, QCD correction should be taken into account when $\chi_{1}^{0}$ dominantly annihilates into the $t \bar{t}$ final state.

Since the dark matter density has been determined with an accuracy of $O(10 \%)$ by WMAP and other experiments, this fact has important implications for future studies of supersymmetric dark matter(s). Once properties of superparticles are determined in future collider experiments, it will become possible to theoretically calculate the relic density of the lightest neutralino (if it is the LSP). Comparison of the result of such a calculation with the observed density of the dark matter will be a crucial test of the scenario of the lightest-neutralino dark matter. In addition, even if the lightest neutralino is not the LSP, precise determination of the freeze-out density of the lightest neutralino may be important for understanding the origin of the dark matter. In particular, possibilities of very weakly interacting LSP (like gravitino-, axino-, or right-handed-sneutrino-LSP), which are called superWIMPs, have been intensively considered recently. If one of the superWIMPs is the LSP, the lightest neutralino becomes unstable. In this case, however, the lifetime of the lightest neutralino is usually very long and hence, if $\chi_{1}^{0}$ is the lightest superparticle in the MSSM sector, thermally produced lightest neutralinos decay after they freeze out from the thermal bath. If this is the case, thermally produced lightest neutralino becomes one of the significant sources of the superWIMP cold dark matter. Thus, even in the scenario with superWIMPs, it is important to calculate the relic density of the lightest neutralino.

Acknowledgements: The authors would like to thank T. Asaka, K. Hikasa and M. Tanabashi for useful discussion and valuable comments. 


\section{References}

[1] WMAP webpage, http://lambda.gsfc.nasa.gov.

[2] T. Moroi, Y. Shimizu and A. Yotsuyanagi, Phys. Lett. B 625, 79 (2005); T. Moroi and Y. Shimizu, Phys. Rev. D 72, 115012 (2005); E. A. Baltz, M. Battaglia, M. E. Peskin and T. Wizansky, arXiv:hep-ph/0602187.

[3] J. R. Ellis, K. A. Olive, Y. Santoso and V. C. Spanos, Phys. Lett. B 565, 176 (2003);

H. Baer and C. Balazs, JCAP 0305, 006 (2003); U. Chattopadhyay, A. Corsetti and P. Nath, Phys. Rev. D 68, 035005 (2003).

[4] V. Barger, W. Y. Keung, H. E. Logan, G. Shaughnessy and A. Tregre, Phys. Lett. B 633, 98 (2006).

[5] J. L. Feng and T. Moroi, Phys. Rev. D 61, 095004 (2000); J. L. Feng, K. T. Matchev and T. Moroi, Phys. Rev. Lett. 84, 2322 (2000); Phys. Rev. D 61, 075005 (2000).

[6] J. L. Feng, K. T. Matchev and F. Wilczek, Phys. Lett. B 482, 388 (2000).

[7] M. Ibe, T. Moroi and T. Yanagida, Phys. Lett. B 620, 9 (2005).

[8] See, for example, A. H. Hoang et al., Eur. Phys. J. directC 2, 1 (2000), and references therein.

[9] T. Moroi, H. Murayama and M. Yamaguchi, Phys. Lett. B 303, 289 (1993); J. L. Feng, A. Rajaraman and F. Takayama, Phys. Rev. Lett. 91, 011302 (2003).

[10] L. Covi, J. E. Kim and L. Roszkowski, Phys. Rev. Lett. 82, 4180 (1999).

[11] T. Asaka, K. Ishiwata and T. Moroi, Phys. Rev. D 73, 051301 (2006).

[12] J. F. Gunion, H. E. Haber, G. L. Kane and S. Dawson, "The Higgs Hunter's Guide," (1990, Addison-Wesley Publishing).

[13] K. i. Hikasa and J. Hisano, Phys. Rev. D 54, 1908 (1996).

[14] M. Drees and K. i. Hikasa, Phys. Lett. B 240, 455 (1990) [Erratum-ibid. B 262, 497 (1991)].

[15] W. Fischler, Nucl. Phys. B 129, 157 (1977).

[16] E. W. Kolb and M. S. Turner, "The Early Universe," (1990, Addison-Wesley Publishing).

[17] B. C. Allanach, G. Belanger, F. Boudjema and A. Pukhov, JHEP 0412, 020 (2004). 